\title{
手指关节可独立控制的欠驱动灵巧手*
}

\author{
刘今越 ${ }^{1,2}$ 赵 点 ${ }^{1,2,3}$ 贾晓辉 1,2 郭士杰 ${ }^{1,2}$ 武晓峰 ${ }^{3}$ 顾建军 ${ }^{3}$
}

(1. 河北工业大学机械工程学院 天津 300130;

2. 河北工业大学河北省机器人感知与人机融合重点实验室 天津 300130 ;

3. 上海航天控制技术研究所 上海 201109)

摘要: 为了实现对物体的精准抓取, 针对欠驱动灵巧手手指关节转动不可控问题提出一种新颖的关节可锁紧的单腱欠驱动灵 巧手的设计方案，完成了灵巧手关节锁紧机构和指间耦合机构的结构设计，并运用 Matlab 对灵巧手手指工作空间进行仿真。 对关节锁紧机构和指间耦合机构动作起到关键作用的耦合弹簧和复位弹簧进行了设计, 对影响关节驱动力矩的参数优化分 析, 得到优化后的手指传动机构和腱绳张紧机构。通过灵巧手样机对不同形状、大小物体的抓握试验, 证明本欠驱动灵巧手 可实现手指关节锁紧的动作, 具有良好的包络抓握能力和较强的精确抓取能力。

关键词: 欠驱动; 精确抓取; 关节锁紧; 灵巧手; 优化设计

中图分类号: TP23

\section{Underactuated Dexterous Hand with Independent Control of Finger Joint}

\author{
LIU Jinyue $^{1,2}$ ZHAO Rui ${ }^{1,2,3}$ JIA Xiaohui ${ }^{1,2}$ GUO Shijie ${ }^{1,2}$ WU Xiaofeng ${ }^{3}$ GU Jianjun ${ }^{3}$
}

(1. School of Mechanical Engineering, Hebei University of Technology, Tianjin 300130;

2. Key Laboratory of Robotic Perception and Human-Machine Integration in Hebei

Province, Hebei University of Technology, Tianjin 300130;

3. Shanghai Institute of Aerospace Control Technology, Shanghai 201109)

\begin{abstract}
In order to achieve accurate grasping of objects, a novel joint-lockable single-turn under-actuated dexterous hand was designed for the uncontrollable problem of under-actuated dexterous hand joint movement. The structural designs of the joint locking mechanism and the inter-finger coupling mechanism were completed, and the working space of the dexterous finger were analyzed with Matlab. The coupling spring and the return spring, which play a key role in the joint locking mechanism and the inter-finger coupling mechanism, were designed to optimize the parameters affecting the joint driving torque, and the optimized finger transmission mechanism and the reel tensioning mechanism are obtained. The trials to grip different shapes and sizes objects by the dexterous hand prototype were carried out to validate the ablilities of locking the finger joints, the envelope grasping and the precision grasping.
\end{abstract}

Key words: underactuation; precise grasp; joint locking; dexterous hand; optimal design

\section{0 前言}

人手是大自然创造出的最完美的工具，在仿生 机器人领域，仿生灵巧手是一个很有价值的研究领 域。其与传统机器人末端夹持器相比, 具有多个多 关节手指, 能够和人手一样具有很高的灵巧性、通 用性和适应性, 能够适应多种抓取和操作任务, 随

* 国家重点研发计划(2017YFB1301002)、河北省自然科学基金 (E2017202270) 和河北省教育厅重点项目 (ZD2018246) 资助项目。 20190530 收到初稿, 20190925 收到修改稿
着灵巧手应用领域的扩大，已逐渐能够替代人手完 成一些复杂的任务, 具有重大的社会应用价值 ${ }^{[1]}$ 。 当前具有拟人特征的灵巧手多采用全驱动形式, 如 Shadow Dexterous Hand ${ }^{[2]} 、$ Robonaut $2^{[3]}$ 等，虽然能 完成物体抓取和一些特定的操作任务，但成本高昂 且控制系统复杂，很难得到广泛应用。而欠驱动灵 巧手结构相对简单、驱动单元少、成本低廉、控制 简单，因此在灵巧手领域应用更为广泛，但是由于 驱动单元个数低于机构自由度个数, 传统欠驱动灵 巧手手指不能独立控制各关节转动，物体抓取过程 中手指位姿不确定，因此只能完成对物体的简单包 
络抓握 ${ }^{[4]}$, 即在驱动机构牵引下, 灵巧手各手指指 节和手掌与物体表面产生自适应接触从而将物体包 络起来, 而并不能实现对物体的精确抓取 ${ }^{[5]}$, 因此 抓取适应性差、对形状复杂的物体抓取稳定性差。 通常来讲, 精确抓取包括指尖捏取、指腹对捏、侧 捏等几种抓取方式 ${ }^{[6]}$ 。与包络抓握不同的是, 精确 抓取时物体与手指的接触区域不同, 接触面积变小, 因而机器人灵巧手在进行精确抓取时难度更高, 对 抓取的稳定程度要求更严格。

为了改善灵巧手精确抓取能力, 从灵巧手的机 构及传动设计出发, 通过对手指结构的创新, 设计 了一种腱绳传动、手指关节转角可控的新型欠驱动 灵巧手, 并对其关键参数进行了设计和优化。该灵 巧手不仅可以很好完成基本包络抓取, 还可以完成 较复杂的指尖抓取，具有结构简单、控制方便、抓 取过程可控等特点。

\section{1 灵巧手机构及传动设计}

\section{1 灵巧手构型设计}

本灵巧手为一种五指灵巧手，其结构如图 1 所 示, 每根手指均有三个转动自由度分别对应三个关 节，整手共五根手指，15 个自由度。

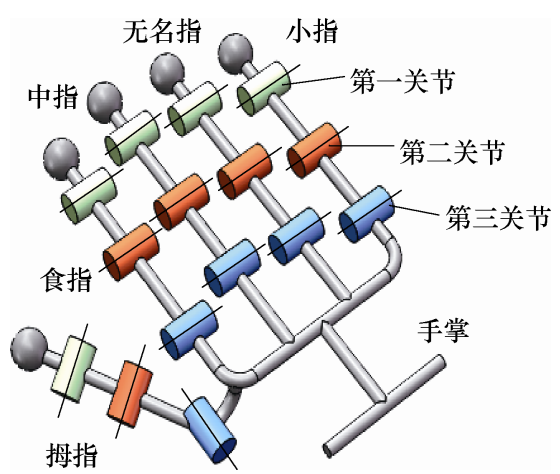

图 1 灵巧手结构简图

\section{2 灵巧手传动方案设计}

本灵巧手采用腱绳-滑轮传动形式, 滑轮布置在 两指节关节处, 腱绳一端与指尖固连, 另一端依次 按规律穿过各滑轮, 最后与驱动电动机连接以提供 拉力。在各关节靠近手背位置开有小孔, 弹性绳从 中穿过提供手指复位所需的恢复力。

图 2 为本灵巧手的单指传动方案设计, 各参数 含义如下: $T$ 为腱绳张力; $R_{i}$ 为各关节滑轮半径; $m_{i}$ 为各指节质量; $l_{i}$ 为各指节长度; $d_{i}$ 为各指节重心 相对各关节的距离; $\theta_{i}$ 为各指节的相对转角。

为了方便手指的空间布置, 在保证运动灵活性 又不使结构因自由度过多而复杂化的前提下, 本灵 巧手基关节位置未设置侧摆自由度, 虽然在仿人性

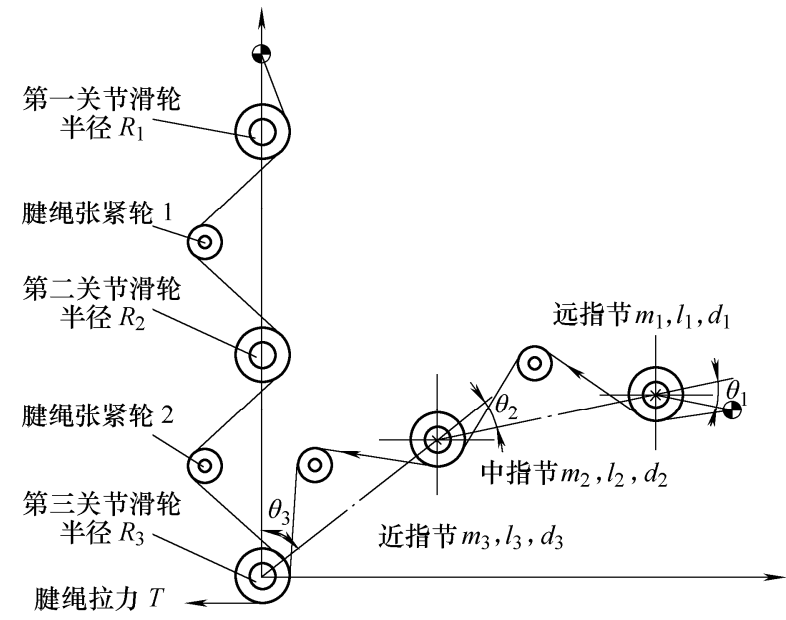

图 2 灵巧手单指传动设计方案

上有所下降，但并未影响其抓取功能的实现; 此外 在拇指的设计上也进行了简化，人类的拇指共有三 个关节，五个自由度，拇指对人手功能的实现是至 关重要的 ${ }^{[7]}$, 但考虑到本研究的重点并不是拇指的 构型, 故将其自由度数简化为三个, 其中为了使拇 指的运动规律尽可能与人手拇指运动规律相近, 令 拇指基关节与手掌夹角约为 $50^{\circ}$ ，与人手相似，拇 指基关节轴线与其余两关节轴线成空间异面直线。

\section{2 灵巧手结构}

\section{1 食指整体结构}

经上述简化，灵巧手各手指的弯曲运动均可视 为具有三个转动自由度的开链连杆平面运动, 除拇 指之外，其他四指(食指、中指、无名指、小指)的 手指构型一致, 另外, 为了实现设计的模块化和互 换性, 令此四指各部分结构参数亦分别相同, 下文 以食指的机构设计为例进行说明, 图 3 为食指结构 示意图。

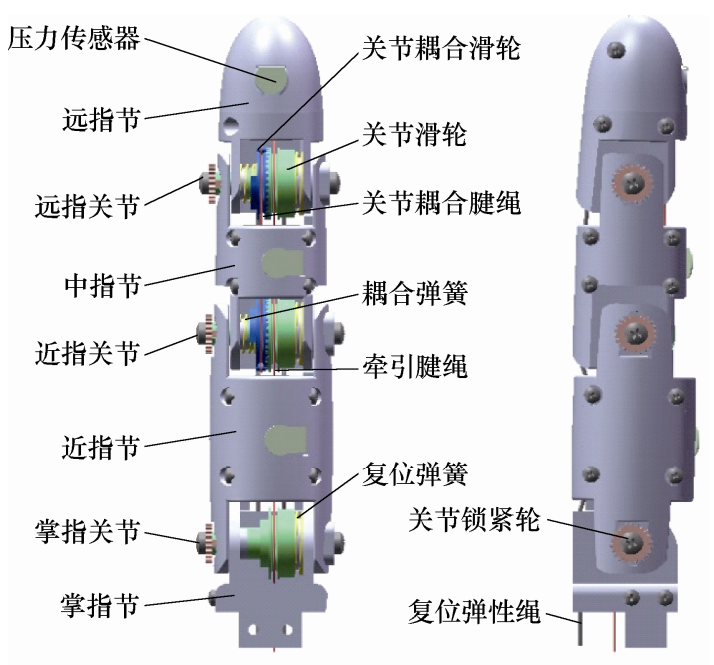

(a) 食指正视图

(b) 食指左视图

图 3 食指结构图 
本灵巧手的核心特点是具有一种关节锁紧机构 使指节转到目标角度时可以停止指节间的相对转动 达到锁死的效果, 基于这样的设计本灵巧手可完成 相对复杂的指尖抓取; 此外, 生物学研究发现, 人 手手指远指关节和近指关节进行屈曲运动的过程中 两关节转角之间的比例近似 $1: 1^{[8]}$, 根据这一特点, 在远指关节和近指关节处添加耦合滑轮, 两个耦合 滑轮通过耦合腱绳连接, 使两关节在非锁死状态下 保持耦合运动关系。

\section{2 食指关节锁紧机构及耦合机构}

食指三个关节均设置相同的关节锁紧机构, 其 工作原理类似于滑键, 因掌指关节没有耦合运动关 系, 构件较少, 故以掌指关节为例说明锁紧机构的 工作原理, 其爆炸图如图 4 所示。

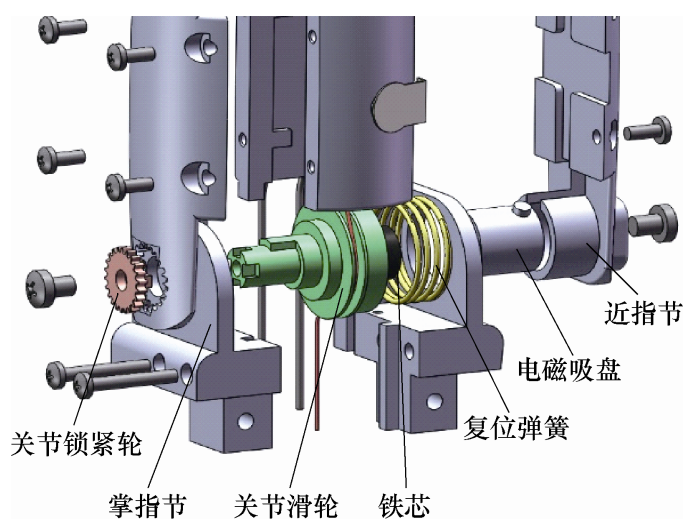

图 4 关节锁紧机构

其具体装配关系为: 关节轴与滑轮为一体式结 构称为关节滑轮, 铁芯镶嵌在关节滑轮右端的凹槽 内, 复位弹簧设置于同一侧的环形槽内, 电磁吸盘 位于复位弹簧右侧, 通过螺钉与近关节连接; 关节 锁紧轮位于关节滑轮左侧末端, 两者通过螺钉相连。

其工作原理与过程为: 关节滑轮左端同时穿过 近指节和掌指节, 并与掌指节通过键连接, 由于掌 指节固定不转动, 故关节滑轮只能左右滑动; 在复 位弹簧的作用下, 关节滑轮移至最左侧, 关节锁紧 轮与近指节之间存在间隙, 近指节和掌指节可相对 转动; 当近指节相对于掌指节转过一定角度后电磁 吸盘通电, 吸引铁芯带动关节滑轮向其移动, 锁紧 轮轮齿与近指节所开齿槽啮合, 近指节与掌指节无 法继续相对转动, 关节锁紧。图 5 为关节锁紧前后 的变化情况。

目前, 实现关节耦合运动的方式主要有三种: “ $\infty$ ” 字形腱绳耦合 ${ }^{[9]}$ 、四连杆机构耦合以及槽型耦 合机构。其中 “ $\infty$ ” 字型腱绳耦合方式将两根交叉 腱的四个固定点分别固定于远指节和近指节, 多用 于远指关节和近指关节存在耦合运动关系的情况, 并未涉及中指节运动耦合问题。而四连杆机构耦合

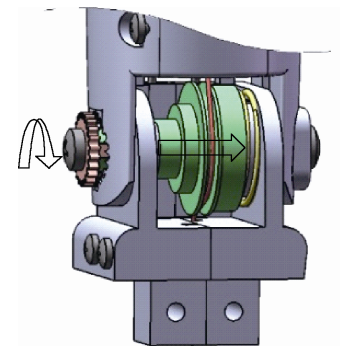

(a) 关节锁紧前

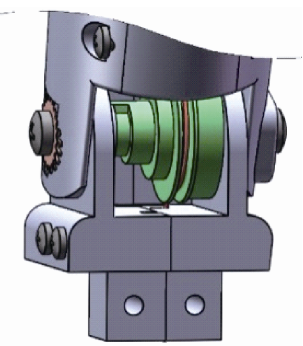

(b) 关节锁紧后

图 5 关节锁紧工作过程

方式不仅在布置时受手指外形尺寸限制而且四杆机 构本身存在死点位置, 在该位置附近机构运动具有 不确定性, 这在灵巧手手指的运动中是不允许的。 相较于前两种机构, 本灵巧手所采用的槽型耦合传 动机构中, 两根腱绳的固定点均分别设置在两个关 节耦合滑轮上, 在需要进行耦合传动时, 耦合滑轮 通过轮齿啮合与关节轴结合在一起同步转动; 当需 要解耦时, 耦合滑轮与关节轴相互分离, 形成一种 可解耦的关节耦合传动方式, 如图 6 所示。
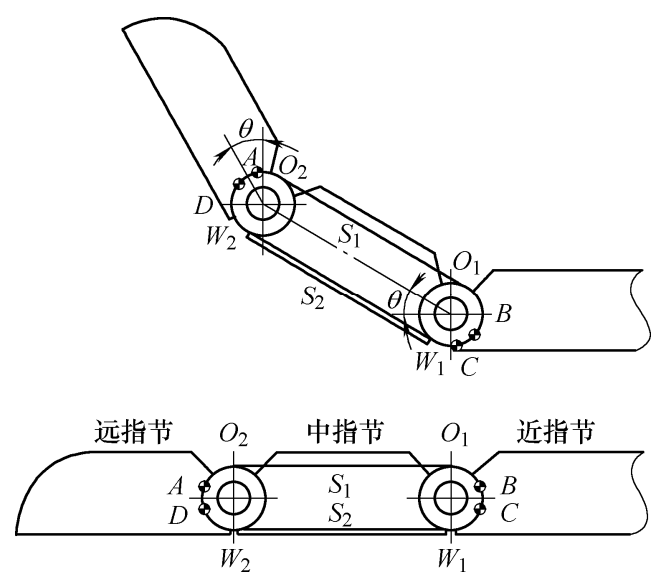

图 6 槽型耦合传动

由图 6 可知, 两根耦合腱绳绕过第一、二关节 的耦合轮 $W_{1} 、 W_{2}$ 将两个关节耦合, 第一关节与第 二关节的传动比 $i$ 为

$$
i=\frac{R^{\prime}}{R}
$$

式中, $R^{\prime}$ 为耦合轮 $W_{2}$ 的半径 $(\mathrm{mm}) ; R$ 为耦合轮 $W_{1}$ 的半径 $(\mathrm{mm})$ 。为保证传动比 $i=1: 1$, 令: $R=R^{\prime}$ 。

之所以将耦合腱绳的固定点均设置在关节耦合 滑轮上, 是因为当手指未接触到物体时, 手指三个 关节在腱绳的牵引下做屈曲运动, 此时远指关节与 近指关节保持耦合运动关系; 当手指与物体接触, 远指关节与近指关节同时或其中一个关节需要锁 紧, 此时应将耦合关系断开, 否则会发生功能上的 冲突。据此, 设计了一种巧妙的可解耦的指问耦合 机构，其工作过程如图 7 所示。 


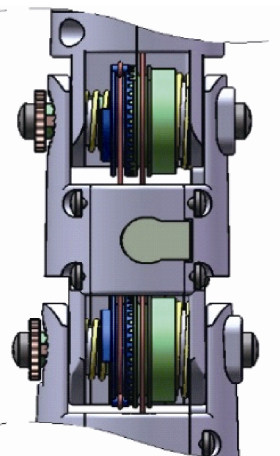

(a) 耦合滑轮与滑轮轴啮合

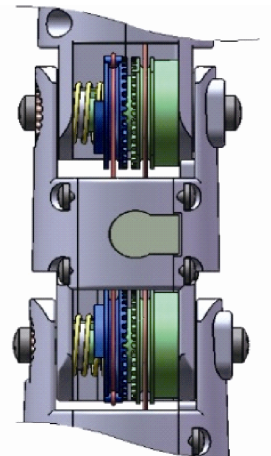

(b) 耦合滑轮与滑轮轴分离

图 7 耦合与耦合关系解除

关节未锁紧时(图 7a), 受右侧弹簧作用, 耦合轮 右端的轮齿与滑轮轴左端的轮齿相互压紧, 发生啮 合, 左侧弹簧被压紧, 两关节产生耦合运动关系; 当 电磁吸盘通电吸引滑轮轴右移关节锁紧时(图 7b), 被 压缩的左侧弹簧放松, 耦合轮与滑轮轴之间产生间 隙，二者轮齿脱离啮合，两关节间的耦合关系解除。

此外, 因本灵巧手中的滑轮轴可左右滑移, 腱 绳亦随之移动, 故分别在食指中指节和近指节内侧 的适当位置设置腱绳松紧调节装置, 如图 8 所示。 所述电磁吸盘实物如图 9 所示。

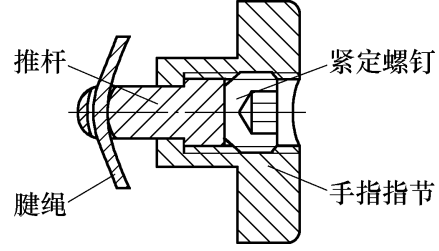

图 8 腱绳松紧调节装置

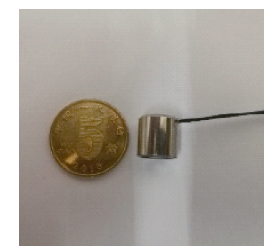

图 9 电磁吸盘实物

手指工作空间仿真可以直观地表征灵巧手手指 运动能力 ${ }^{[10-11]}$ 。因此, 完成单根手指结构设计后, 采用蒙特卡洛方法 ${ }^{[12]}$ 对食指工作空间进行分析。

在已知各指节长度的前提下，以各关节转角 $\theta_{i}(i=1 、 2 、 3)$ 为变量, 在 $\theta_{i}$ 取值范围 $(0 \sim \pi / 2)$ 内对 其随机取一组值, 则指尖参考点对应一个随机空 间位置, 通过取多组 $\theta_{i}$ 值得到指尖参考点全部随 机位置组成的点云空间 ${ }^{[13]}$ 即为手指的工作空间。 利用 Matlab 软件结合手指正运动学方程, 随机生 成 50000 组 $\theta_{i}$ 值, 仿真出了食指工作空间如图 10 所示。

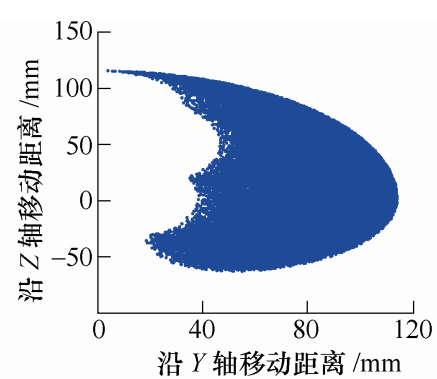

(a) $O y z$ 平面

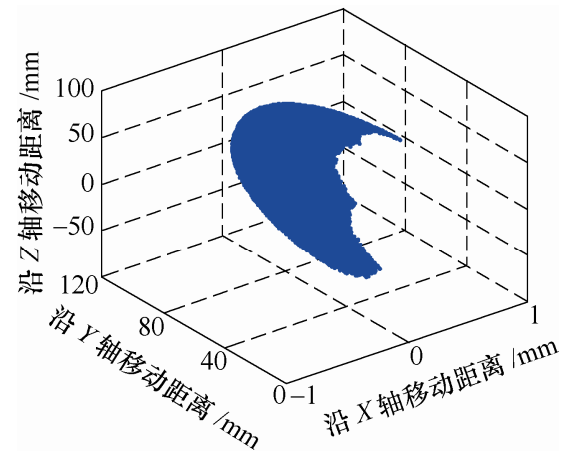

(b) $O x y z$ 三维空间

图 10 食指工作空间

\section{3 拇指整体结构}

在抓取和操作任务中拇指的作用不可替代，人 手能实现的绝大部分功能都有拇指的参与, 如精确 抓取、捏拿、包络抓取等。

拇指比其他四指更加灵活, 自由度个数更多, 设计较为困难。根据人手生理学研究结果, 将拇指 掌指关节转动自由度的回转轴线设计为近似坚直位 置，与其余坚直方向布置的四指呈一定夹角，同时 考虑人手拇指的对掌运动 ${ }^{[14]}$, 设计拇指近指关节轴 线与掌指关节轴线呈空间异面直线，两者夹角 ${ }^{[15]}$ 为 $35^{\circ}$, 这样可以尽可能地模拟人手的对掌运动, 在简 化拇指设计的同时又不失拇指的拟人性，最后远指 关节轴线和近指关节轴线平行，这样就完成了对拇 指自由度在空间上的布置。

拇指驱动传动方式和其余四指相同，考虑拇指 的中指节和远指节并无耦合运动关系, 故在拇指远 指关节和近指关节处不设置耦合传动机构。

具体拇指结构形式上，在远指关节和近指关节处 添加关节锁紧机构; 掌指关节处不设置关节锁紧机 构, 一方面是因为当拇指与物体接触时, 若远指关节 和近指关节的转角确定，掌指关节的转角也可以确定 下来, 所以拇指掌指关节的转动只是为抓取物体提供 夹紧力; 另一方面则是与掌指关节自由度在空间上的 特殊布局有关，即其回转轴线与坚直方向有一定的夹 角, 若布置关节锁紧机构难度较大, 且因元件重力影 响易使机构动作灵活性和准确度降低。根据以上考 虑, 设计的拇指三维结构如图 11 所示。

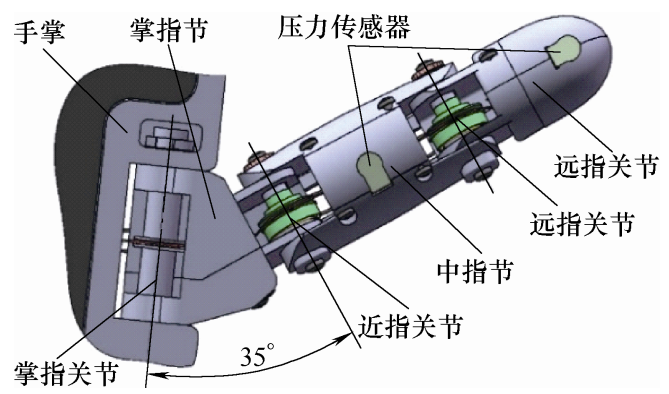

图 11 拇指三维结构图 
同理, 用分析食指工作空间的方法分析拇指工 作空间, 随机生成 50000 组关节角度 $\theta_{i}$, 得到的拇 指工作空间如图 12 所示。

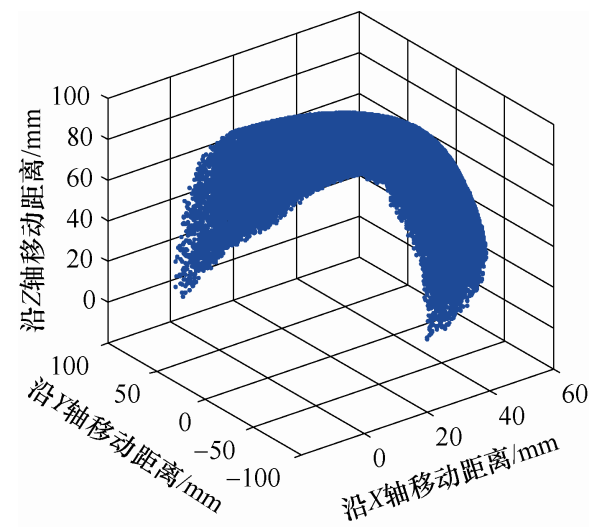

图 12 拇指工作空间仿真

\section{4 灵巧手的整体结构}

与人手相似, 该机器人灵巧手设计 5 根手指, 共使用 5 个电动机作为驱动源。各手指尺寸参考人 手手指长度特征设计; 为了方便模块化设计和满足 互换性要求, 设计食指、中指、无名指、小指四指 结构相同; 所有手指均安装在手掌上, 手掌及驱动 电动机安装在手臂上, 集成化程度较高。

表 1 给出各手指参数。在各指节设置 FSR 400 型压力传感器, 检测抓握力大小。灵巧手样机如 图 13 所示。

表 1 手指参数

\begin{tabular}{cccc}
\hline 名称 & 指节长度 $/ \mathrm{mm}$ & 手指总长 $/ \mathrm{mm}$ & 各关节转角 $/\left({ }^{\circ}\right)$ \\
\hline 食指 & $31.5,33.0,49.5$ & 114 & $0 \sim 90$ \\
中指 & $31.5,33.0,49.5$ & 114 & $0 \sim 90$ \\
无名指 & $31.5,33.0,49.5$ & 114 & $0 \sim 90$ \\
小指 & $31.5,33.0,49.5$ & 114 & $0 \sim 90$ \\
拇指 & $37.5,50.0,23.5$ & 111 & $0 \sim 90$ \\
\hline
\end{tabular}

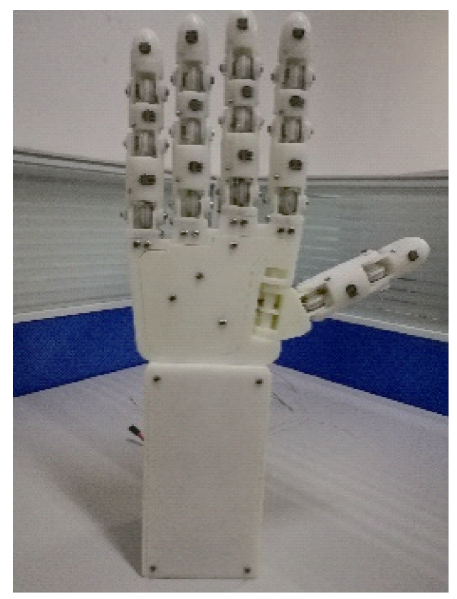

图 13 灵巧手样机

\section{3 欠驱动灵巧手关键参数分析}

\section{1 弹簧参数分析}

手指关节锁紧和指节耦合运动功能的实现主要 依靠电磁吸盘吸引力和左右两侧弹簧的弹性复位 力, 所以弹簧参数 ${ }^{[16]}$ 的设计与选取至关重要。

除拇指之外，其他四指的远指节和中指节之间在 关节未锁紧时存在耦合运动关系, 将与耦合轮配合使 用的弹簧称为耦合弹簧, 将关节锁紧后提供滑轮轴 复位力的弹簧称为复位弹簧; 耦合弹簧和复位弹簧 在关节锁紧机构中的两种工作状态如图 14 所示。

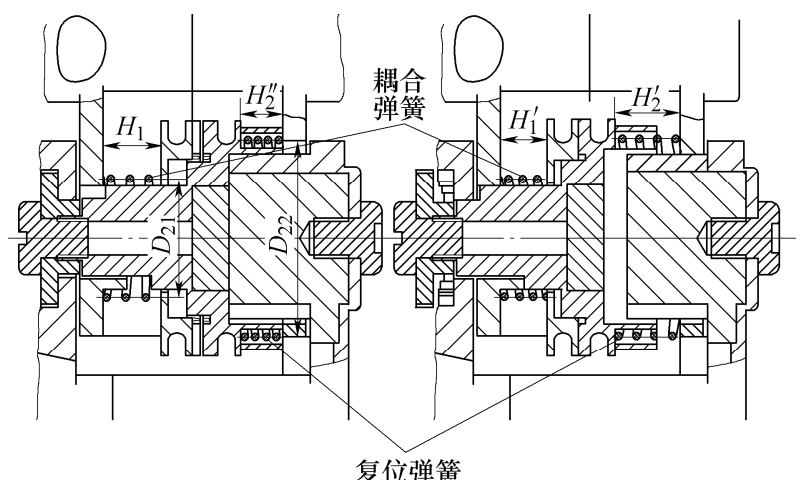

(a) 关节锁紧弹簧状态

(b) 关节未锁紧弹簧状态

图 14 弹簧工作状态

耦合弹簧的工作状态为: 假设弹簧原长为 $H_{1}$, 当远指关节和近指关节的关节锁紧机构均未动作 时, 关节滑轮轴位于最左端位置, 与耦合滑轮处于 贴合状态, 弹簧被压缩, 长度为 $H_{1}{ }^{\prime}$, 变形量为 $\lambda_{1}$, 弹簧力为 $F_{1}$, 两关节间存在耦合运动关系, 如图 $14 \mathrm{~b}$ 所示。

当远指关节和近指关节中任意一个锁紧时, 关 节滑轮轴被拉到最右端, 耦合弹簧放松至原长 $H_{1}$, 耦合滑轮和关节滑轮轴相互脱离, 运动互不干涉, 耦合关系解除，如图 14a 所示。

复位弹簧的具体工作过程为: 假设弹簧原长为 $\mathrm{H}_{2}$, 在关节锁紧机构未动作时, 弹簧处于压缩状态, 长度被压缩为 $H_{2}{ }^{\prime}$, 变形量为 $\lambda_{2}$, 产生的弹簧力为 $F_{2}$, 在 $F_{2}$ 的作用下关节滑轮轴被推至手指关节内侧 的最左端, 锁紧轮与下部指节外侧的轮齿槽脱开, 关节滑轮轴通过键传动随上部指节转动与下部指节 互不干涉, 手指关节可以自由转动, 如图 $14 \mathrm{~b}$ 所示。

若电磁铁通电, 关节锁紧机构产生动作, 关节 滑轮轴被吸引至关节内侧最右端, 在此过程中, 复 位弹簧被进一步压缩, 长度为 $H_{2}{ }^{\prime \prime}$, 变形量为 $\lambda_{2}{ }^{\prime}$, 弹簧力为 $F_{2}{ }^{\prime}$, 此时锁紧轮与下部关节外侧齿槽啮 合, 使上下两指节转角固定; 在动作结束时电磁铁 
断电, 关节滑轮轴在复位力 $F_{2}{ }^{\prime}$ 作用下移动至关节最 左端, 如图 14a 所示。在整个过程中, 复位弹簧始 终处于被压缩状态。

根据上述弹簧工作过程分析, 总结两处弹簧的 设计原则: 在关节耦合状态时, 复位弹簧内力应大 于耦合弹簧内力; 在关节解耦状态时, 复位弹簧内 力小于电磁铁吸引力 $F_{\mathrm{a}}$; 各弹簧内径应大于与其相 配合部分的关节滑轮轴轴径。

弹簧刚度

$$
k=\frac{F}{\lambda}=\frac{G d}{8 C^{3} n}=\frac{G d^{4}}{8\left(D_{1}+d\right)^{3} n}
$$

式中, 旋绕比 $C=D_{2} / d, d$ 为簧丝直径, 中径 $D_{2}=D_{1}+d$, 内径 $D_{1}$, 外径 $D$, 圈数 $n$, 弹簧材料的切变模量 $G$ 。

耦合弹簧簧丝直径为 $d_{1}$, 中径 $D_{2}{ }^{1}$, 内径 $D_{1}{ }^{1}$, 外径 $D^{1}$, 圈数 $n_{1}$; 复位弹簧簧丝直径 $d_{2}$, 中径 $D_{2}{ }^{2}$, 内径 $D_{1}{ }^{2}$, 外径 $D^{2}$, 圈数 $n_{2}$ 。

耦合状态: $H-H_{1}^{\prime}=\lambda_{1}, H_{2}-H_{2}^{\prime}=\lambda_{2}, F_{1}=\lambda_{1} k_{1}$, $F_{2}=\lambda_{2} k_{2}, \quad F_{1} \leqslant F_{2}$ 。

锁紧状态: $\lambda_{1}{ }^{\prime}=0, H_{2}-H_{2}{ }^{\prime \prime}=\lambda_{2}{ }^{\prime}, F_{1}{ }^{\prime}=0, F_{2}{ }^{\prime}=\lambda_{2}{ }^{\prime} k_{2}$, $F_{2}{ }^{\prime}<F_{\text {a }}$ 。

由式(2)可得两种弹簧刚度为

$$
\left\{\begin{array}{l}
k_{1}=\frac{G d_{1}^{4}}{8 D_{2}^{1^{3}} n_{1}} \\
k_{2}=\frac{G d_{2}{ }^{4}}{8 D_{2}^{2^{3}} n_{2}}
\end{array}\right.
$$

弹簧长度 $H_{1} 、 H_{1}{ }^{\prime} 、 H_{2}{ }^{\prime} 、 H_{2}{ }^{\prime \prime}$ 均已知, 根据实际情 况设计耦合弹簧 $D_{2}{ }^{1}=9 \mathrm{~mm} 、 d_{1}=0.4 \mathrm{~mm} 、 n_{1}=3 、 H_{1}=5$ $\mathrm{mm}$; 设计复位弹簧 $D_{2}^{2}=14 \mathrm{~mm} 、 d_{2}=0.8 \mathrm{~mm} 、 n_{2}=3$ 、 $H_{2}=7 \mathrm{~mm}$, 由式(3)可得: $k_{1}=0.35 \mathrm{~N} / \mathrm{mm}, k_{2}=0.49 \mathrm{~N} / \mathrm{mm}$ 。

考虑到复位弹簧所处的位置离电磁铁较近, 为 避免其工作受电磁铁影响, 故选不锈钢作为复位弹 簧的材料, 耦合弹簧对材料无特殊要求, 故采用价 格较低廉的碳素弹簧钢。图 15 和图 16 分别为加工 出的耦合弹簧和复位弹簧实物。

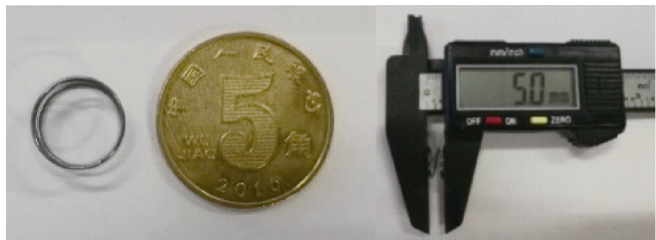

图 15 耦合弹簧实物

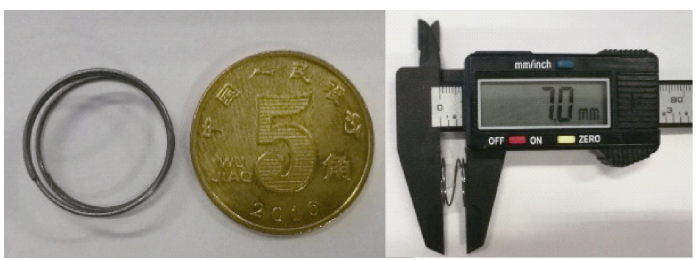

图 16 复位弹簧实物

\section{2 影响关节驱动力矩的参数优化分析}

以食指的传动机构为例, 手指是通过单根腱绳 和滑轮实现运动的传递的, 在近指节和中指节处设 置有腱绳松紧调节装置, 抓取动作受传动机构的影 响较大。在传动机构中滑轮的尺寸和形式是影响手 指出力的关键，在相同腱绳张力的情况下不同的传 动机构对各关节的驱动转矩是不同的, 因此最佳的 状态是在相同腱拉力条件下，使手指关节获得最大 的驱动力矩, 这是进行滑轮参数优化的重要依据。

为了方便分析, 将腱绳松紧调节装置推杆末端 简化成一小滑轮，食指传动形式简化成图 17 。

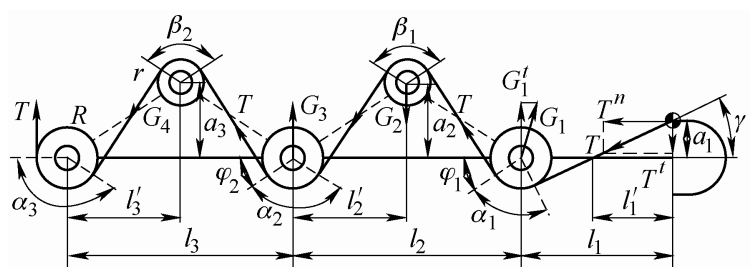

图 17 食指传动形式简化图

其中手指的几何参数分别如下: $l_{1} 、 l_{2} 、 l_{3}$ 分别 为远指节、指节、近指节长度; $a_{1}$ 为腱绳指尖结点 的偏心距; $a_{2} 、 a_{3}$ 分别为两小滑轮到对应指节的距 离; $l_{2}^{\prime} 、 l_{3}^{\prime}$ 分别为小滑轮到对应关节的距离; $r$ 为 指节小滑轮半径， $R$ 为关节滑轮半径; $\alpha_{1} 、 \alpha_{2} 、 \alpha_{3}$ 分别为腱绳在关节滑轮上的包角; $\beta_{1} 、 \beta_{2}$ 分别为腱 绳在小滑轮上的包角; $\varphi_{1} 、 \varphi_{2} 、 \gamma$ 分别为食指相关结 构角, 与几何参数有关。

手指的受力情况为: $T$ 为腱绳牵引力; $T^{t}$ 为牵 引力切向分力; $T^{n}$ 为牵引力法向分力; $G_{i}(i=1,2,3$, 4)为腱绳对各滑轮的等效力; $G_{i}^{t}$ 为等效力的切向分 力, 由于图示的几何关系, 导致 $G_{2}^{t} 、 G_{4}^{t}$ 与 $G_{2} 、 G_{4}$ 完全相等，在图中不予表示。

令手指在牵引力 $T$ 作用下转动, 如图 18 所示, 其中, $\theta_{1}, \theta_{2}, \theta_{3}$ 分别为各指节转角, $\alpha_{1}^{\prime}, \alpha_{2}^{\prime}, \alpha_{3}^{\prime}$ 分别为 手指转动后腱绳在关节滑轮上的包角; $\beta_{1}^{\prime}, \beta_{2}^{\prime}$ 分别 为手指转动后腱绳在小滑轮上的包角; $G_{i}^{\prime}(i=1,2,3$, 4)为手指转动后腱绳对各滑轮的等效力; $G_{i}^{\prime \prime}$ 为对应 等效力的切向分力。

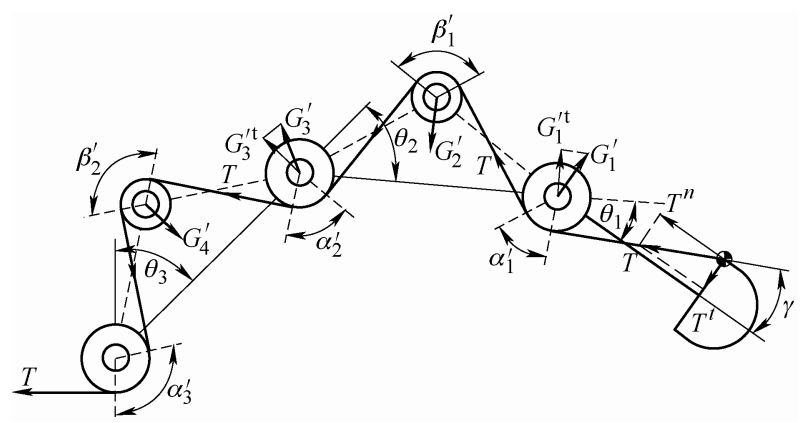

图 18 食指运动后受力图 
以下将依次对远指关节、近指关节、掌指关节 的驱动力矩进行分析。

对远指关节进行受力分析, 得到远指关节的驱 动力矩为

$$
M_{1}=M_{1}^{\prime}-M_{1}^{\prime \prime}=T R
$$

式中, $M_{1}^{\prime}=T^{t} l_{1}, M_{1}^{\prime \prime}=T^{n} a_{1}, T^{t}=T \sin \gamma$, $T^{n}=T \cos \gamma \quad, \quad l_{1}=l_{1}^{\prime}+l_{1}^{\prime \prime} \quad, \quad \tan \beta_{1}=a_{1} / l_{1}^{\prime}$, $\sin \beta_{1}=R / l_{1}^{\prime \prime}$ 。

由式(4)可知, 在腱绳牵引力 $T$ 和远指节长度 $l_{1}$ 固定不变的情况下, 远指关节驱动力矩 $M_{1}$ 主要受关 节滑轮半径 $R$ 影响, 与其他参数无关, 所以应在保 证实际结构空间的前提下, 尽可能增加关节滑轮半 径 $R$ 以增加驱动力矩。

假设近指关节的驱动力矩为 $M_{2}$, 分析其受力 可知

$$
M_{2}=M_{2}^{\prime}-M_{2}^{\prime \prime}+M_{2}^{\prime \prime \prime}
$$

式中, $M_{2}^{\prime}$ 为腱绳力 $G_{2}^{\prime}$ 产生的力矩, $M_{2}^{\prime \prime}$ 为腱绳力 $G_{1}^{\prime}$ 产生的力矩, $M_{2}^{\prime \prime \prime}$ 为指尖汼引力 $T$ 产生的力矩。

由式(5)可知, 要得到足够大的力矩 $M_{2}$ 则应使 $M_{2}^{\prime}$ 和 $M_{2}^{\prime \prime}$ 尽可能大, 而 $M_{2}^{\prime \prime}$ 尽可能小。由于食指发 生转动后模型上各参考点发生较大变化, 若对每个 力矩均进行定量分析则过于复杂, 因此, 将对 $M_{2}^{\prime}$ 和 $M_{2}^{\prime \prime}$ 进行定量分析, 而对 $M_{2}^{\prime \prime}$ 进行定性分析, 以达 到增加驱动力的效果。

对于 $M_{2}^{\prime}$, 有如下关系式

$$
\left\{\begin{array}{l}
M_{2}^{\prime}=G_{2}^{\prime} l_{2}^{\prime} \\
G_{2}^{\prime}=2 T \cos \frac{\pi-\beta_{1}^{\prime}}{2} \\
\beta_{1}^{\prime}=\beta_{1}
\end{array}\right.
$$

将式(6)化简可得

$$
M_{2}^{\prime}=2 T l_{2}^{\prime} \sin \frac{\beta_{1}}{2}
$$

由式(7)可知, 若腱绳牵引力 $T$ 不变, 那么力矩 $M_{2}^{\prime}$ 的 大小取决于 $\beta_{1} \in(0 \sim \pi)$ 和 $l_{2}^{\prime}$ 的取值。由食指结构可 知, 增加小滑轮半径 $r$, 则可使夹角 $\beta_{1}$ 变大; 或者 改变小滑轮位置使其更靠近掌指关节则可使间距 $l_{2}^{\prime}$ 增大, 这两种方法均可以得到较大的力矩 $M_{2}^{\prime}$ 。

对于 $M_{2}^{\prime \prime}$, 有如下关系式

$$
\left\{\begin{array}{l}
M_{2}^{\prime \prime}=G_{1}^{\prime} l_{2} \sin \left(\varphi_{1}+\frac{\alpha_{1}^{\prime}}{2}\right) \\
\alpha_{1}-\alpha_{1}^{\prime}=\theta_{1} \\
G_{1}^{\prime}=2 T \cos \frac{\pi-\alpha_{1}^{\prime}}{2}
\end{array}\right.
$$

将式(8)化简可得

$$
M_{2}^{\prime \prime}=2 T l_{2} \sin \left(\varphi_{1}+\frac{\alpha_{1}-\theta_{1}}{2}\right) \sin \left(\frac{\alpha_{1}-\theta_{1}}{2}\right)
$$

分析式(9)可知, $M_{2}^{\prime \prime}$ 为阻力矩, 若已经对远指关节 滑轮进行了优化, 那么夹角 $\alpha_{1}$ 为固定值, 要使力矩 $M_{2}^{\prime \prime}$ 尽可能小, 则应当适度减小结构角 $\varphi_{1}$, 增加小 滑轮到指节的距离 $a_{2}$ 或小滑轮半径 $r$, 即, 使小滑 轮更偏离指节同时增大小滑轮。

对于 $M_{2}^{\prime \prime \prime}$, 在关节转动初始阶段, $M_{2}^{\prime \prime}$ 为正向 驱动力矩; 当关节转动至腱绳汼引力 $T$ 的延长线经 过近指关节中心点时, 对应的驱动力臂为零, $M_{2}^{\prime \prime \prime}$ 减 小为零; 随着关节进一步转动, $M_{2}^{\prime \prime}$ 继续减小进而 方向发生改变, 变为负向力矩, 对关节转动形成阻 碍, 而且在整个转动过程中, $M_{2}^{\prime \prime}$ 始终遵循此规律, 因此应尽量避免此类情况出现。经分析可知, 增大 结构角 $\gamma$ 可使 $M_{2}^{\prime \prime}$ 变为负力矩的过程延后, 所以应 适度增大关节滑轮半径 $R$, 与之前得出的结论相同。

综上所述, 在腱绳牵引力 $T$ 不变的情况下, 若 要使近指关节驱动力矩 $M_{2}$ 增加, 应适度增大小滑 轮半径 $r$ 且使其远离(两个方向)近指关节中心。同理 分析掌指关节驱动力矩, 所得结论与近指关节相同, 这里不再赘述。优化后食指传动方案如图 19 中虚线 所示。

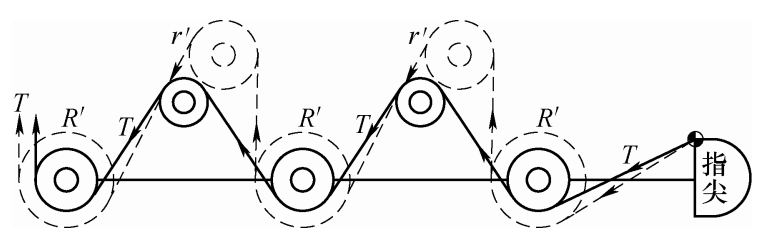

图 19 滑轮单元的受力分析与几何关系

除此之外, 优化过程中, 尚需注意可能出现的 摩擦力增加、手指尺寸粗大等问题, 综合考虑结构 实现的可能性、零件加工和装配的简便性等因素, 合理设置滑轮各参数。

\section{4 灵巧手样机试验}

灵巧手的主要功能是物体抓取, 本灵巧手手指 关节处添加了关节锁紧机构, 因此, 在关节锁紧机 构不动作时, 本灵巧手可对物体完成较简单的包络 抓握; 在关节锁紧机构动作时, 可以完成更为复杂 的精确抓取。本节对灵巧手的两种抓取模式进行试 验验证。

本灵巧手手指材料为光敏树脂, 各手指远指节 表面硬度较大且光滑, 因此, 试验时在各手指指尖 包覆橡胶材料, 增加手指与物体之间的摩擦力。 


\section{1 灵巧手包络抓握试验}

灵巧手在进行包络抓握时, 五根手指均向掌内 运动, 各关节锁紧机构均不起作用, 五个手指在电 动机的㴆引下动作, 将物体包围并握紧, 完成包络 抓握。图 20 所示为方形饮料瓶、圆形饮料瓶和体型 较小的工具瓶的包络抓握试验。

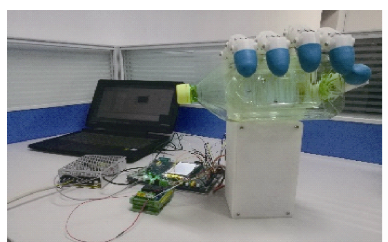

(a) 抓取方形饮料瓶

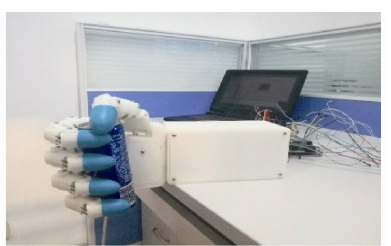

(b) 抓取圆形饮料瓶

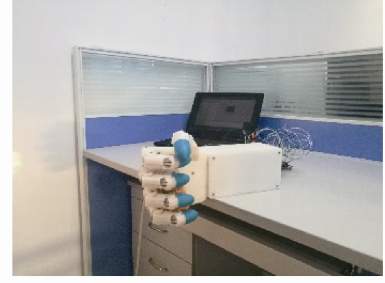

(c) 抓取圆形工具瓶

图 20 包络抓握试验

试验结果表明, 本灵巧手对不同大小和形状的 物体具有较强的包络抓握能力, 适应性较强且能保 持稳定抓握，不使物体滑落。

\section{2 灵巧手精确抓取试验}

灵巧手在进行精确抓取时需要关节止转机构的 配合, 实现手指位姿在空间的固定。以精确抓取中 的典型方式一指尖对捏来进行精确抓取试验, 以食 指和拇指为对象验证灵巧手的精确抓取性能。图 21 所示分别为灵巧手对瓶盖、线圈、铌子、螺丝刀等 物体进行指尖对捏试验。

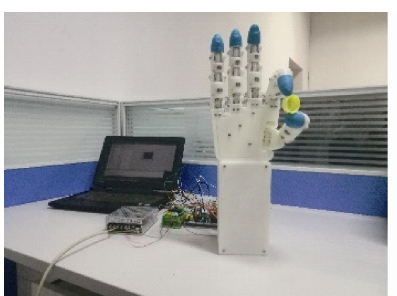

(a) 抓取瓶盖

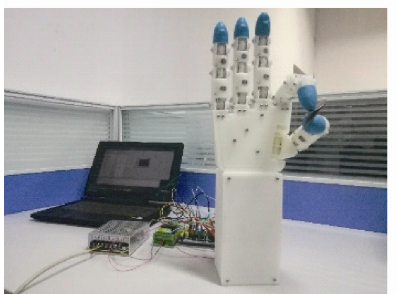

(c) 抓取镊子

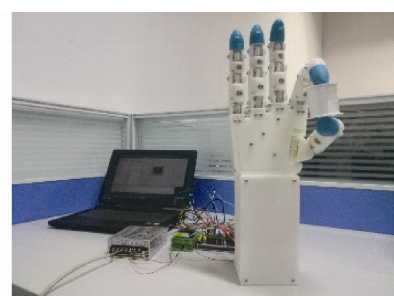

(b) 抓取线圈

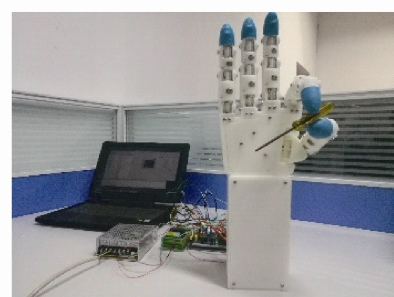

(d) 抓取螺丝刀
图 21 指尖对捏试验

试验结果表明, 本灵巧手的关节止转机构动作 可行, 工作可靠，手指具有对不同形状的小物体进
行精确抓取的能力，抓握稳定性较好。

\section{5 结论}

(1) 相较于传统欠驱动灵巧手而言, 本欠驱动 灵巧手在手指关节处添加了用于控制关节转动角 度的关节锁紧机构, 使得每个关节的转动变得可 控, 并且运用模块化设计思想, 将除拇指外的四指 设计为相同结构, 增加了互换性, 方便了手指加工 和组装。

（2）本灵巧手所设计的关节锁紧机构的动作受 弹簧的影响很大，在充分分析了手指和关节锁紧机 构的工作过程后, 确定了弹簧的设计原则, 经过计 算得到了耦合弹簧和复位弹簧的机构参数并制作了 弹簧实物。

（3）通过抓握试验, 验证了本灵巧手样机不仅 可以进行简单的包络抓握, 还可以通过关节锁紧机 构控制各指节关节转角, 完成复杂的精确抓取, 抓 取性能良好。

总之, 通过对传统欠驱动灵巧手进行结构上的 改进, 添加了关节锁紧机构, 在不增加电动机驱动 的情况下，使欠驱动灵巧手指可以独立控制每个关 节的转角, 达到了全驱动灵巧手指的效果, 这是一 种创新性的尝试, 是未来灵巧手设计的一个很重要 的方向。

\section{参 考 文 献}

[1] 刘菲, 何广平, 陆震. 弹性欠驱动四指灵巧手设计与试 验 $[\mathrm{J}$. 机械工程学报，2014，50(15): 53-59.

LIU Fei, HE Guangping, LU Zhen. Research and design of a novel tendon driven underactuated multi-fingered hand[J]. Journal of Mechanical Engineering, 2014, 50(15): 53-59.

[2] KOCHAN A. Shadow delivers first hand[J]. Industrial Robot: An International Journal, 2005, 32(1): 15-16.

[3] BAKER W, KINGSTON Z, MOLL M, et al. Robonaut 2 and you : Specifying and executing complex operations[C]// 2017 IEEE Workshop on Advanced Robotics and its Social Impacts (ARSO), September 4, 2017, Austin, TX, USA: IEEE, 2017: 1-8.

[4] NAPIER J R. The prehensile movements of the human hand[J]. The Journal of Bone and Joint Surgery-British Volume, 1956, 38(4): 902-913.

[5] 李剑锋, 张玉茹, 张启先. 多指手的操作灵巧性设计 [J]. 机械工程学报, 2000，36(11): 23-29.

LI Jianfeng, ZHANG Yuru, ZHANG Qixian. Dexterity design of multi-fingered hand[J]. Chinese Journal of 
Mechanical Engineering, 2000, 36(11): 23-29.

[6] HAMILTON N, LUTTGENS K. Kinesiology: Scientific basis of human motion[M]. McGraw-Hill Higher Education, 2002.

[7] 王海荣. 仿人型灵巧手拇指灵巧性设计方法的研究 [D]. 哈尔滨: 哈尔滨工业大学, 2012.

WANG Hairong. Research on the dexterity design method of humanoid dexterous hand[D]. Harbin: Harbin Institute of Technology, 2012.

[8] ARBUCKLE J D, MCGROUTHER D A. Measurement of the arc of digital flexion and joint movement ranges[J]. The Journal of Hand Surgery British \& European Volume, 1996, 20(6): 836-840.

[9] 㚞绍巍, 刘伊威, 金明河, 等. HIT/DLR Hand II 类人形 五指灵巧手机构的研究 [J]. 哈尔滨工程大学学报, 2009, 30(2): 171-177.

FAN Shaowei, LIU Yiwei, JIN Minghe, et al. Research on the mechanics of the HIT/DLR Hand II anthropomorphic five-finger dexterous hand[J]. Journal of Harbin Engineering University, 2009，30(2): 171-177.

[10] 李剑锋, 张凯, 张雷雨, 等. 并联踝康复机器人的设计 与运动性能评价[J]. 机械工程学报, 2019, 55(9): 29-39. LI Jianfeng, ZHANG Kai, ZHANG Leiyu, et al. Design and kinematic performance evaluation of parallel ankle rehabilitation robot[J]. Journal of Mechanical Engineering, 2019, 55(9): 29-39.

[11] 赵燕江, 张永德, 姜金刚, 等. 基于 Matlab 的机器人 工作空间求解方法 [J]. 机械科学与技术, 2009, 28(12): 1657-1661.

ZHAO Yanjiang, ZHANG Yongde, JIANG Jingang, et al. A method for solving robot workspace based on Matlab[J]. Mechanical Science and Technology for Aerospace
Engineering, 2009, 28(12): 1657-1661.

[12] DIAO Xiumin, MA Ou. Workspace analysis of a 6-DOF cable robot for hardware-in-the-loop dynamic simulation[C]// 2006 IEEE/RSJ International Conference on Intelligent Robots \& Systems, Oct. 9-15, 2006, Beijing, China: IEEE, 2006: 4103-4108.

[13] FU Jianxun, GAO Feng. Optimal design of a 3-leg 6-DOF parallel manipulator for a specific workspace[J]. Chinese Journal of Mechanical Engineering, 2016, 30(4): 659-668.

[14] 韦酋亨, 赵卫东, 谢扬, 等. 拇指对掌运动 6 个自由度 的采集与分析[J]. 中国临床解剖学杂志, 2012, 30(2): 229-232.

WEI Qiuheng, ZHAO Weidong, XIE Yang, et al. Collection and analysis of 6 degrees of freedom of thumb opposition movement[J]. Chinese Journal of Clinical Anatomy, 2012, 30(2): 229-232.

[15] 刘伊威, 金明河, 㚞绍巍, 等. 五指仿人机器人灵巧手 DLR/HIT Hand II[J]. 机械工程学报, 2009，45(11): 16-23.

LIU Yiwei, JIN Minghe, FAN Shaowei, et al. Five-finger dextrous robot hand DLR/HIT hand II[J]. Journal of Mechanical Engineering, 2009, 45(11): 16-23.

[16] SUN Jianjun. Investigation into effect of spring pressure on performance of balanced mechanical seals[J]. Chinese Journal of Mechanical Engineering, 2007, 20(3): 39-43.

作者简介: 刘今越, 男, 1977 年出生, 博士, 教授, 硕士研究生导师。 主要研究方向为智能机器人、智能检测与控制。

E-mail: 1jy@hebut.edu.cn

赵睿, 男, 1993 年出生, 硕士研究生。主要研究方向为机器人技术及应用。 E-mail: zr199366@163.com 贾晓辉(通信作者), 女, 1976 年出生, 博士, 讲师。主要研究方向为力 触觉感知、柔性精密定位系统设计。

E-mail: jia_dragon@163.com 